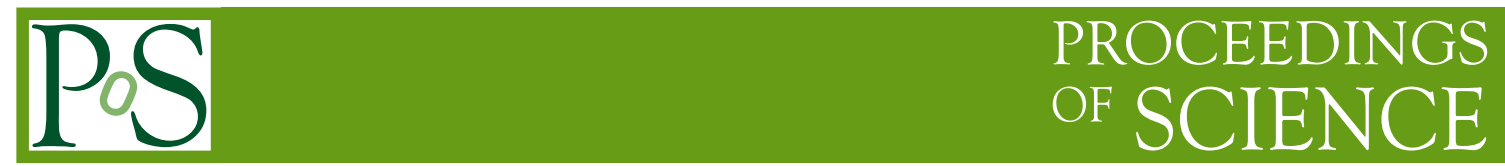

\title{
Unintegrated parton densities at small $x$
}

\author{
Martin Hentschinski*i \\ Instituto de Física Teórica UAM/CSIC, Universidad Autónoma de Madrid, Cantoblanco, \\ E-28049 Madrid, Spain \\ E-mail: martin.hentschinski@uam.es
}

We present recent results on transverse momentum dependent parton densities in the limit of small Bjorken $x$. A definition of an unintegrated seaquark density is proposed which can be used within the CCFM Monte-Carlo event generator CASCADE. We further discuss aspects of the determination of a NLO BFKL unintegrated gluon density.

The 2011 Europhysics Conference on High Energy Physics-HEP 2011,

July 21-27, 2011

Grenoble, Rhône-Alpes France

\footnotetext{
* Speaker.

${ }^{\dagger}$ Work in collaboration with F. Hautmann, H. Jung, A. Sabio Vera and C. Salas Hernandez
} 


\section{Introduction}

Transverse momentum dependent parton distribution arise naturally at small $x$ as a consequence of high energy factorization and BFKL-evolution [1]. A formulation of high energy factorization which is in accordance with conventional collinear factorization is provided by $k_{T}$ factorization as defined in [2,3]: at high center of mass energies, the resummation of high energy logarithms (BFKL) can be brought into a form consistent with conventional collinear resummation (DGLAP). In the following we present ongoing work dedicated to the study of transverse momentum dependent parton densities at small $x$. In sec. 2 we address the definition of an unintegrated sea-quark for the CASCADE Monte Carlo event generator. In sec. 3 we discuss the determination of a transverse momentum dependent gluon density which takes into account full next-to-leading order BFKL resummation.

\section{A $k_{T}$-dependent sea-quark density for the CASCADE}

The Monte Carlo event generator CASCADE [4] is based on the CCFM evolution equation [5]. The latter interpolates between DGLAP and BFKL evolution and thus allows naturally for a Monte Carlo implementation of $k_{T}$-factorization. Based on the principle of color coherence, the CCFM parton shower describes at first only the emission of gluons, while emissions of quarks are left aside. This is justified as enhanced regions of phase space for $x \rightarrow 1$ and $x \rightarrow 0$ are dominated by gluonic dynamics at leading logarithmic level. As far as unintegrated parton densities are concerned, this implies that CCFM evolution describes only the distinct evolution of unintegrated gluon and valence quarks while transitions between quarks and gluons are left aside. Although formally sub-leading, quark emissions can give give numerical sizeable contributions and it is therefore advisable to include them into account in the parton shower, too. Absence of quark emissions affects furthermore the determination of $k_{T}$-dependent hard matrix elements: due to absence of a seaquark density, the gluon-to-quark splitting needs to be included into matrix element which complicates their determination, see e.g. [6]. As a first step towards arriving at a complete inclusion of quark emission into the CCFM parton shower, we present here a definition of an off-shell sea-quark density and a partonic matrix element. For the former we restrict to the case where the gluon-to-quark splitting occurs as the last evolution step. For a detailed discussion we refer to [7].

For definiteness we consider in the following the transverse momentum dependent factorization of the $q g^{*} \rightarrow q Z$ matrix element into a transverse momentum dependent partonic sub-process $q q^{*} \rightarrow Z$ and a transverse momentum dependent quark-gluon splitting function. The latter is given by the e $k_{T}$-dependent quark-gluon splitting function of [3] which reads

$$
P_{q g}\left(z, \frac{\mathbf{k}^{2}}{\mathbf{q}^{2}}\right)=T_{R}\left(\frac{\mathbf{q}^{2}}{\mathbf{q}^{2}+z(1-z) \mathbf{k}^{2}}\right)^{2}\left[(1-z)^{2}+z^{2}+4 z^{2}(1-z)^{2} \frac{\mathbf{k}^{2}}{\mathbf{q}^{2}}\right],
$$

Here $\mathbf{k}$ and $\mathbf{q}$ denote the transverse momentum of the off-shell gluon and off-shell quark respectively, while $z$ denotes the fraction of gluon light cone momentum which is carried on by the $t$ channel quark. Note that in the on-shell limit $\mathbf{k}^{2} \rightarrow 0$ eq. (2.1) reduces to the conventional DGLAP splitting function $T_{R}\left[z^{2}+(1-z)^{2}\right]$. For the determination of the $q q^{*} \rightarrow Z$ coefficient we follow closely the treatment of the already existing gluonic case. There off-shell gauge invariance is ensured through a reformulation of QCD at high center of mass energies in terms of effective degrees 
of freedom, reggeized gluons. The latter coincide in their on-shell limit with conventional collinear QCD gluons. For the general off-shell case one uses effective vertices which contain additional induced terms, which ensure off-shell gauge invariance ${ }^{1}$. In complete analogy one can construct a reggeized quark formalism for the description of the high energy limit of scattering amplitudes with quark exchange in the $t$-channel [9]. As (reggeized) quark exchanges are in comparison to (reggeized) gluon exchanges suppressed by powers of $s$, they generally do not occur in the high energy resummation of total cross-sections. They can however be used as a starting point for the construction of an off-shell factorization of matrix elements which are limited to quark exchange in the $t$-channel. This allows to define the off-shell partonic cross-section $q q^{*} \rightarrow Z$ as the analytic continuation of the $q q \rightarrow Z$ cross-section, in complete analogy to the gluonic case. The splitting function on the other hand is within this approach at first obtained as a pure constant, corresponding to the limit $z=0$ of eq. (2.1). It is however possible to incorporate finite $z$-corrections into the reggeized quark effective vertices, which allow to re-obtain eq. (2.1) for the gluon-to-quark splitting. Combination of eq. (2.1) with the unintegrated gluon density then yields the unintegrated quark density

$$
\mathscr{Q}^{\text {sea }}\left(x, \mathbf{q}^{2}, \mu^{2}\right)=\frac{1}{\mathbf{q}^{2}} \int_{x}^{1} \frac{d z}{z} \int_{0}^{\mathbf{k}_{\max }^{2}} d \mathbf{k}^{2} \frac{\alpha_{s}\left(\mu^{2}\right)}{2 \pi} P_{q g}\left(z, \frac{\mathbf{k}^{2}}{\mathbf{q}^{2}}\right) \mathscr{G}\left(\frac{x}{z}, \mathbf{k}^{2}, \bar{\mu}^{2}\right) .
$$

with $\mathbf{k}_{\max }^{2}=\mu^{2} / z-\mathbf{q}^{2} /(z(1-z))$. For further details and numerical results we refer to [7].

\section{NLO BFKL gluon density}

We determine a transverse momentum dependent gluon density which follows NLO BFKL evolution. It is obtained as the convolution

$$
\mathscr{G}\left(x, \boldsymbol{q}^{2}\right)=\int \frac{d^{2} \boldsymbol{k}}{\pi} f_{\mathrm{BFKL}}(x, \boldsymbol{q}, \boldsymbol{k}) \Phi_{P}\left(\boldsymbol{k}^{2}\right) .
$$

with NLO BFKL Green's function $f_{\mathrm{BFKL}}$ and a model for the proton impact factor

$$
\Phi_{P}\left(\boldsymbol{k}^{2}, Q_{0}^{2}, \delta, A\right)=A \frac{1}{\boldsymbol{k}^{2}}\left(\frac{\boldsymbol{k}^{2}}{Q_{0}^{2}}\right)^{\delta} e^{-\frac{k^{2}}{Q_{0}^{2}}}
$$

with free parameters $\left\{Q_{0}, A, \delta\right\}$ obtained from a fit to HERA data. The $F_{2}$ structure function requires to convolute the gluon density with the photon impact factor, where we use the LO kinematical improved impact factor of [11]. Given the recent progress in the determination of NLO corrections to effective vertices of the gluonic effective action [10], it seems however possible to obtain NLO impact factors for future studies of DIS and and LHC cross-sections. The NLO BFKL Green's function is known to possess a numerical instability due to the presence of (anti-) collinear logarithms. For the present study the latter are resummed using an extension of the 'allpole'-approximation of [12]. It has the advantage that it allows for an exponentiation of the BFKL kernel, in agreement with Regge-theory. Furthermore a transformation from transverse moment to

\footnotetext{
${ }^{1}$ For an effective action approach to the reggeized gluon formalism see [8].
} 
transverse momentum space is possible in that case. This is of importance as it allows to implement this Green's function into a NLO BFKL Monte Carlo event generator [13] which is currently investigated. For a detailed discussion and the results of the fit we refer to [14]

\section{Acknowledgments}

I wish to thank my collaborators F. Hautmann, H Jung, A. Sabio Vera and C. Salas Hernandez for intense and numerous discussions. Financial support from the German Academic Exchange Service (DAAD), the MICINN under grant FPA2010-17747 and the Research Executive Agency (REA) of the European Union under the Grant Agreement number PITN-GA-2010-264564 (LHCPhenoNet) is gratefully acknowledged.

\section{References}

[1] L. N. Lipatov, Sov. J. Nucl. Phys. 23 (1976) 338 [Yad. Fiz. 23 (1976) 642]; V. S. Fadin, E. A. Kuraev and L. N. Lipatov, Phys. Lett. B 60 (1975) 50; I. I. Balitsky and L. N. Lipatov, Sov. J. Nucl. Phys. 28 (1978) 822 [Yad. Fiz. 28 (1978) 1597]. V. S. Fadin and L. N. Lipatov, Phys. Lett. B 429 (1998) 127 [hep-ph/9802290].

[2] S. Catani, M. Ciafaloni and F. Hautmann, Phys. Lett. B 307 (1993) 147; Nucl. Phys. B 366 (1991) 135; Phys. Lett. B242 (1990) 97.

[3] S. Catani and F. Hautmann, Nucl. Phys. B 427 (1994) 475; Phys. Lett. B315 (1993) 157.

[4] H. Jung and G. P. Salam, Eur. Phys. J. C 19 (2001) 351 [arXiv:hep-ph/0012143]; H. Jung et al., Eur. Phys. J. C 70, 1237 (2010) [arXiv:1008.0152 [hep-ph]].

[5] M. Ciafaloni, Nucl. Phys. B296 (1988) 49, S. Catani, F. Fiorani, G. Marchesini, Phys. Lett. B234 (1990) 339, S. Catani, F. Fiorani, G. Marchesini, Nucl. Phys. B336 (1990) 18, G. Marchesini, Nucl. Phys. B445 (1995) 49-80. [hep-ph/9412327].

[6] M. Deak and F. Schwennsen, JHEP 0809 (2008) 035 [arXiv:0805.3763 [hep-ph]], S. P. Baranov, A. V. Lipatov and N. P. Zotov, Phys. Rev. D 78 (2008) 014025 [arXiv:0805.4821 [hep-ph]].

[7] F. Hautmann, M. Hentschinski, H. Jung, in preparation.

[8] L. N. Lipatov, Nucl. Phys. B452 (1995) 369-400 [arXiv:hep-ph/9502308 [hep-ph]]; L. N. Lipatov, Phys. Rept. 286 (1997) 131-198 [hep-ph/9610276]; M. Hentschinski, [arXiv:0908.2576 [hep-ph]]; M. Hentschinski, Nucl. Phys. Proc. Suppl. 198 (2010) 108-111 [arXiv:0910.2981 [hep-ph]].

[9] L. N. Lipatov and M. I. Vyazovsky, Nucl. Phys. B 597 (2001) 399 [arXiv:hep-ph/0009340]; A. V. Bogdan and V. S. Fadin, Nucl. Phys. B 740, 36 (2006) [arXiv:hep-ph/0601117]; V. A. Saleev, Phys. Rev. D 78, 114031 (2008) [arXiv:0812.0946 [hep-ph]].

[10] M. Hentschinski and A. S. Vera, arXiv:1110.6741 [hep-ph], M. Hentschinski, in preparation G. Chachamis, M. Hentschinski, J. Madrigal Martinez, A. Sabio Vera, in preparation.

[11] A. Bialas, H. Navelet and R. B. Peschanski, Nucl. Phys. B 603 (2001) 218 [hep-ph/0101179].

[12] A. Sabio Vera, Nucl. Phys. B 722 (2005) 65 [hep-ph/0505128].

[13] G. Chachamis, M. Hentschinski, A. Sabio Vera and C. Salas, arXiv:0911.2662 [hep-ph].

G. Chachamis, M. Deak, A. S. Vera and P. Stephens, Nucl. Phys. B 849 (2011) 28 [arXiv:1102.1890 [hep-ph]]. J. R. Andersen and A. Sabio Vera, Nucl. Phys. B 679 (2004) 345 [hep-ph/0309331]. J. R. Andersen and A. Sabio Vera, Phys. Lett. B 567 (2003) 116 [hep-ph/0305236].

[14] M. Hentschinski, A. Sabio Vera and C. Salas, in preparation. 